OPEN ACCESS

Edited by:

Mohammad-Hossein Sarrafzadeh, University of Tehran, Iran

Reviewed by: Hashem Asgharnejad, Polytechnique Montréal, Canada Tanmay Majumdar, National Institute of Immunology (NII), India

*Correspondence: Yang Zhang zhangyang07@hit.edu.cn Junyi Li

lijunyi@hit.edu.cn

Specialty section: This article was submitted to Systems Microbiology, a section of the journal Frontiers in Microbiology

Received: 11 July 2021 Accepted: 30 September 2021 Published: 28 October 2021

Citation:

Zhang $Y, Y e T, X i H$, Juhas $M$ and Li J (2021) Deep Learning Driven Drug Discovery: Tackling Severe Acute Respiratory Syndrome Coronavirus 2. Front. Microbiol. 12:739684. doi: 10.3389/fmicb.2021.739684

\section{Deep Learning Driven Drug Discovery: Tackling Severe Acute Respiratory Syndrome Coronavirus 2}

\author{
Yang Zhang ${ }^{1 *}$, Taoyu Ye ${ }^{1}$, Hui Xi', Mario Juhas ${ }^{2}$ and Junyi Li ${ }^{3 *}$ \\ ${ }^{1}$ College of Science, Harbin Institute of Technology (Shenzhen), Shenzhen, China, ${ }^{2}$ Medical and Molecular Microbiology \\ Unit, Department of Medicine, Faculty of Science and Medicine, University of Fribourg, Fribourg, Switzerland, ${ }^{3}$ School \\ of Computer Science and Technology, Harbin Institute of Technology (Shenzhen), Shenzhen, China
}

Deep learning significantly accelerates the drug discovery process, and contributes to global efforts to stop the spread of infectious diseases. Besides enhancing the efficiency of screening of antimicrobial compounds against a broad spectrum of pathogens, deep learning has also the potential to efficiently and reliably identify drug candidates against Severe Acute Respiratory Syndrome Coronavirus 2 (SARS-CoV2). Consequently, deep learning has been successfully used for the identification of a number of potential drugs against SARS-CoV-2, including Atazanavir, Remdesivir, Kaletra, Enalaprilat, Venetoclax, Posaconazole, Daclatasvir, Ombitasvir, Toremifene, Niclosamide, Dexamethasone, Indomethacin, Pralatrexate, Azithromycin, Palmatine, and Sauchinone. This mini-review discusses recent advances and future perspectives of deep learning-based SARS-CoV-2 drug discovery.

Keywords: deep learning, database, drug discovery, antibiotics, antimalarial drug, drug repurposing, SARS-CoV-2

\section{INTRODUCTION}

Deep learning is a branch of machine learning. It is an algorithm that abstracts data by using multiple processing layers composed of complex structures or multiple non-linear transformations. Compared with the shallow machine learning methods, deep learning algorithm is a process of automatic feature engineering. Deep learning frameworks, such as convolutional neural network and recursive neural network, have been applied in the fields of bioinformatics and biomedicine and achieved excellent results (Lipinski et al., 2019). Deep learning methods have good applications in microbiology including metagenomic data analysis, microbial-related drug discovery, disease microbial association and so on (Duch et al., 2007). When analyzing microbial related data, it shows high prediction accuracy in practice. Because deep learning algorithms are good at obtaining very complex underlying patterns in data, they are especially suitable for large and high-dimensional data sets. Moreover, it is easy to update the model with the new data. The hidden layer of the network obviously reduces the demand for Feature Engineering and is conducive to the completion of prediction tasks. The schematic diagram of the deep learning in drug discovery is shown in Figure 1.

Deep learning has revolutionized most areas of science and technology, including drug discovery. Traditional drug discovery methods are not time and cost efficient and therefore often unable to keep pace with the rapidly emerging and re-emerging pathogenic microorganisms. More recent drug discovery methods include Naive Bayesian, Support Vector Machines and Neural Networks (Bender et al., 2007; Stephenson et al., 2019). These alternative drug discovery 

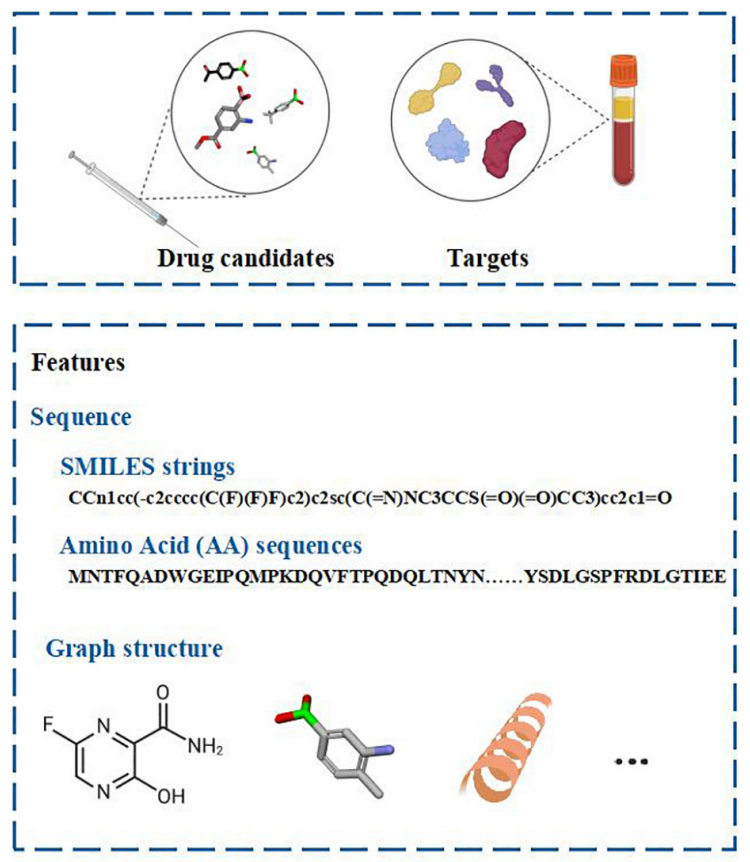

Input dataset

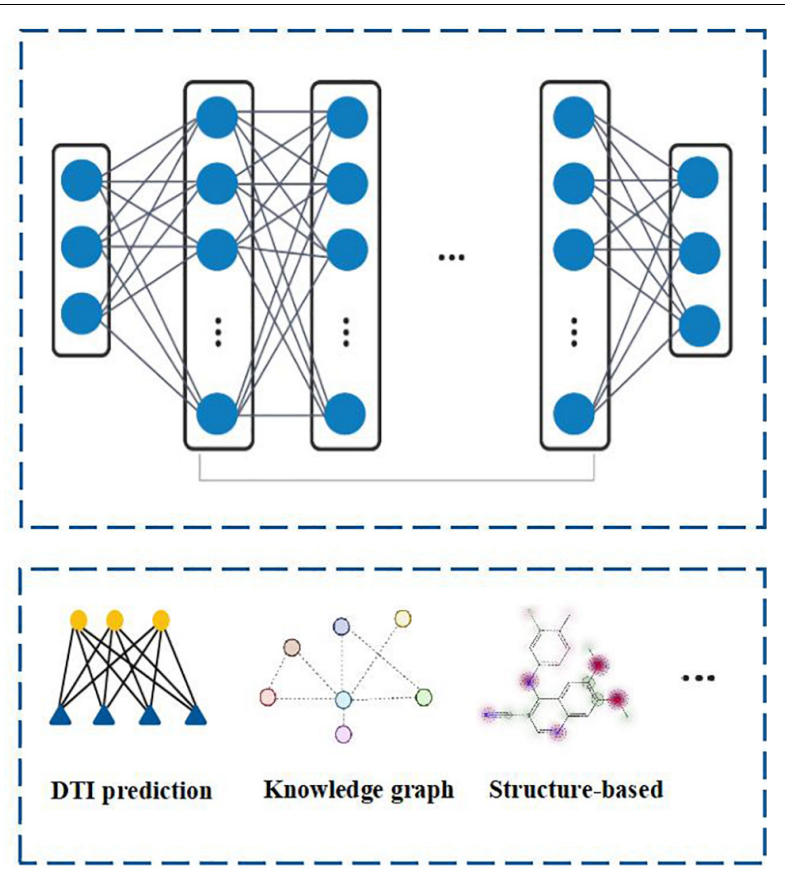

Deep learning models

FIGURE 1 | The schematic diagram of deep learning in drug discovery. Biochemical data from drug candidates and protein targets can be used for drug discovery. Chemical sequences (simplified molecular-input line-entry system (SMILES) strings) and amino acid (AA) sequences, structure of chemical compounds and protein targets can be used as the features to fed into the deep learning models. Different deep learning models can be employed to analyze the data by integrating Drug Target Interaction (DTI) prediction, knowledge graph or structure based computational methods. Figure is made in part with BioRender.

methods usually use bigger data sets generated from high throughput screenings and allow more accurate prediction of bioactivities and molecular properties of the targets (Stephenson et al., 2019). Compared to these alternative machine learning methods used for drug discovery, deep learning is characterized by the flexibility of the architecture of Neural Networks (Chen et al., 2018). Given the cost and time required for traditional drug discovery, deep learning has the potential to significantly accelerate the drug discovery process. By using information on the biological, chemical, and topological properties of compounds and their putative targets from the large-scale libraries, deep learning can be employed to identify the most promising drugs against specific diseases (Neves et al., 2020; Stokes et al., 2020). Various deep learning methods have been developed over the last few years, but their application in drug discovery has still not reached its full potential. One of the main hurdles for researchers planning to build their own deep learning model for drug identification is the amount of resources and time required to collect large amounts of data. A number of computational screen open databases have been made to prioritize drug candidates, recently. A representative set of open access datasets which can be used to train deep learning models for specific research projects is shown in Table $\mathbf{1}$.

The outbreak of severe acute respiratory syndrome coronavirus 2 (SARS-CoV-2) causing coronavirus disease (COVID-19) has been declared a global pandemic. By September 2021, more than 220 million people have been infected with
SARS-CoV-2 and more than 4.5 million of those infected have died. In addition, several SARS-CoV-2 variants with mutations that increase their potential to contribute to the severity of the pandemic have emerged and are spreading around the globe (Zhang Y. et al., 2020).

Besides non-structural proteins, SARS-CoV-2 genome encodes four structural proteins: envelope (E), membrane (M), nucleocapsid (N), and spike (S) (Zhang Y. et al., 2020). S protein mediates entry of SARS-CoV-2 into the host cells by binding and fusing with the host's cellular receptor, the angiotensinconverting enzyme 2 (ACE2). Mutations in S protein, particularly in its receptor binding domain (RBD) were shown to play a role in the increased transmissibility and infectivity of the emerging SARS-CoV-2 variants (Zahradnik et al., 2021).

Although several SARS-CoV-2 vaccines have been developed over the last few months, there are not many efficient and reliable drugs available for the treatment of SARS-CoV2 infections. This is caused partially by the fact that the traditional drug discovery process may be time-consuming and costly to keep pace with the rapid spread of SARS-CoV2 and its variants with increased transmissibility and other enhanced features.

Deep learning has been previously applied for the identification of a number of antiviral compounds, including antiviral peptides (Timmons and Hewage, 2021) and small drug-like compounds with the potential to inhibit HIV-1 (Andrianov et al., 2021). 
The computational approaches employing deep learning will aid also faster discovery of novel and active potential inhibition agents against SARS-CoV-2 and its emerging variants.

High-throughput technologies have generated an increasing amount of data in chemoinformatics. As a result, it is believed that the application of the recent deep learning advances into the drug discovery process will lead to novel, more reliable and efficient therapeutics against SARS-CoV-2.

\section{ANTIMICROBIAL DRUGS IDENTIFIED BY DEEP LEARNING}

Deep learning can reduce time and costs of the drug discovery process, particularly in its early stages. Consequently, deep learning-based approaches have been successfully used to identify novel antimicrobial compounds against a wide variety of pathogenic microorganisms, including bacteria, protozoan parasites and viruses.

Training of the deep learning model to identify molecules active against antibiotic-resistant bacteria led to the discovery of Halicin and eight additional potential antibiotics from the ZINC database (Table 1; Stokes et al., 2020). Interestingly, these compounds identified by deep learning are all structurally divergent from conventional antibiotics (Stokes et al., 2020). Subsequent tests revealed strong antibacterial activity of Halicin against a number of antibiotic-resistant bacteria, including Carbapenemase-producing Enterobacterales, Mycobacterium tuberculosis, Acinetobacter baumannii, and Clostridioides difficile (Stokes et al., 2020).

In parasite research, deep learning has been applied to predict new antimalarial drug candidates. Neves et al. (2020) employed deep learning to obtain binary, continuous Quantitative Structure-Activity Relationships (QSAR) models using datasets extracted from ChEMBL database (Table 1). QSAR mathematical models can predict the relationship between the structure of a molecule and biological activity or physicochemical property. This study led to the discovery of two new families of the potential next generation antimalarial drugs with activity against Plasmodium causing malaria at nanomolar concentrations and low cytotoxicity in mammalian cells (Neves et al., 2020).

Deep learning has been also applied for the identification of a number of antiviral compounds. Timmons and Hewage developed a novel method called ENNAVIA, which employs deep learning and chemoinformatics, to identify peptides with low toxicity and excellent biological activity. The peptides identified in this study represent promising candidates for antiviral drugs (Timmons and Hewage, 2021). Furthermore, deep learning in combination with molecular modeling has been applied for the identification of three small drug-like compounds from millions of molecules in the ZINC15 database (Andrianov et al., 2021). Based on machine learning, molecular docking, molecular dynamics and quantum chemical calculations, the compounds identified in this study are promising HIV-1 drugs with the potential to block CD4-binding site of the viral envelope protein,
TABLE 1 | Representative biochemical datasets used in deep learning studies.

\begin{tabular}{|c|c|c|c|}
\hline Dataset & Description & URL & References \\
\hline ZINC & $\begin{array}{c}\text { ZINC database } \\
\text { contains over } 230 \\
\text { million compounds. }\end{array}$ & http://zinc.docking.org/ & $\begin{array}{l}\text { Bai et al., } \\
\text { 2020; Choi } \\
\text { et al., 2020; } \\
\text { Stokes et al., } \\
\text { 2020; Ton } \\
\text { et al., } 2020\end{array}$ \\
\hline ChEMBL & $\begin{array}{l}\text { ChEMBL (version 27) } \\
\text { chemical database } \\
\text { contains over } 1.9 \\
\text { million specific } \\
\text { compounds. }\end{array}$ & $\begin{array}{c}\text { https://www.ebi.ac.uk/ } \\
\text { chembl/ }\end{array}$ & $\begin{array}{c}\text { Stokes et al., } \\
2020\end{array}$ \\
\hline $\begin{array}{l}\text { Drug target } \\
\text { commons } \\
\text { (DTC) }\end{array}$ & $\begin{array}{c}\text { DTC crowdsourcing } \\
\text { database contains } \\
204,901 \text { annotated } \\
\text { bioactivity data points } \\
\text { among 4,276 } \\
\text { compounds and 1,007 } \\
\text { specific protein targets. }\end{array}$ & $\begin{array}{c}\text { https: } \\
\text { //drugtargetcommons. } \\
\text { fimm.fi/ }\end{array}$ & $\begin{array}{l}\text { Beck et al., } \\
2020\end{array}$ \\
\hline BindingDB & $\begin{array}{l}\text { BindingDB database of } \\
\text { measured binding } \\
\text { affinities contains } \\
2,061,017 \text { binding data } \\
\text { for } 8,160 \text { protein } \\
\text { targets and } 907,259 \\
\text { small molecules. }\end{array}$ & $\begin{array}{l}\text { http://www.bindingdb. } \\
\text { org/bind/index.jsp }\end{array}$ & $\begin{array}{c}\text { Beck et al., } \\
2020\end{array}$ \\
\hline DrugBank & $\begin{array}{c}\text { DrugBank } \\
\text { pharmaceutical } \\
\text { database contains } \\
\text { detailed molecular } \\
\text { information about } \\
\text { drugs, their } \\
\text { mechanisms, } \\
\text { interactions and } \\
\text { targets. }\end{array}$ & $\begin{array}{c}\text { https://go.drugbank. } \\
\text { com/releases/latest }\end{array}$ & $\begin{array}{l}\text { Choi et al., } \\
\text { 2020; Zeng } \\
\text { et al., } 2020\end{array}$ \\
\hline PDBbind & $\begin{array}{l}\text { PDBbind database } \\
\text { provides binding data } \\
\text { of } 21,382 \text { biomolecular } \\
\text { complexes, including } \\
\text { protein-ligand }(17,679) \text {, } \\
\text { nucleic acid-ligand } \\
\text { (136), protein-nucleic } \\
\text { acid (973), and } \\
\text { protein-protein } \\
\text { complexes }(2,594) \text {. }\end{array}$ & $\begin{array}{c}\text { http: } \\
\text { //www.pdbbind.org.cn }\end{array}$ & $\begin{array}{l}\text { Bai et al., } \\
2020\end{array}$ \\
\hline
\end{tabular}

thus inhibiting HIV-1 entry (Andrianov et al., 2021). Li et al. have developed a dual-channel deep neural network for identifying variable-length antiviral peptides (DeepAVP) which could block entry of the virus into the host cell (Li et al., 2020). Deep learning has been also used for the prediction of plant-exclusive virusderived small interfering RNAs (PVsiRNAPred) (He et al., 2019).

\section{DEEP LEARNING IN TACKLING SEVERE ACUTE RESPIRATORY SYNDROME CORONAVIRUS 2}

Reliable and efficient computing methods employing deep learning are urgently needed for the discovery of drugs against SARS-CoV-2 and its emerging variants. 
Drug repurposing is considered to be among the fastest and most promising methods for identification of effective SARSCoV-2 treatments. A good example of the drug repurposing involving deep learning is a recent work by Zhang Y. et al. (2020). This study employed a deep learning-based drug-target interaction model called Molecule Transformer-Drug Target Interaction (MT-DTI) utilizing chemical sequences [simplified molecular-input line-entry system (SMILES) strings] and amino acid (AA) sequences as the input (Figure 1). MT-DTI model was trained with a combined and curated chemical-protein pairs from BindingDB and Drug Target Commons (DTC) databases (Table 1). This study led to identification of several commercially available antiviral drugs with the potential to interact also with the SARS-CoV-2 proteins (Beck et al., 2020). Subsequent experiments showed that several of the antiviral agents identified by MT-DTI model could be potentially used to treat SARS-CoV2 (Beck et al., 2020). These include Atazanavir (Kd $94.94 \mathrm{nM}$ ), Remdesivir (Kd $113.13 \mathrm{nM}$ ), and Kaletra (Lopinavir/Ritonavir) (Table 2). Atazanavir, showing an inhibitory potency against SARS-CoV-2 3-C like proteinase is an antiviral drug used for the treatment of the human immunodeficiency virus (HIV) infections. Remdesivir has been previously predicted to act against SARS-CoV-2. Furthermore, Lopinavir and Ritonavir were shown to target viral proteinases (Beck et al., 2020). The BindingDB is a public database containing measured binding affinities for three types of coronaviruses, SARS-CoV-2, SARS$\mathrm{CoV}$ and MERS-CoV. ${ }^{1}$

MT-DTI model was also used to select compounds from 1,400 approved drugs in DrugBank and ZINC databases (Table 1) with strong affinity to the host cell targets crucial for viral infection (Zahradnik et al., 2021). This approach led to identification of drugs candidates with a strong binding affinity $(\mathrm{Kd}<100 \mathrm{nM})$ against ACE2 receptor and transmembrane protease serine 2 (TMPRSS2) (Zahradnik et al., 2021). Drug candidates identified in this study include an ACE2 inhibitor Enalaprilat (Kd $1.46 \mathrm{nM}$ ) and several drugs with predicted strong affinity for TMPRSS2, namely Venetoclax (Kd $6.12 \mathrm{nM}$ ), Posaconazole (Kd 17.11 $\mathrm{nM})$, Daclatasvir (Kd $6.65 \mathrm{nM}$ ), and Ombitasvir (Kd $5.91 \mathrm{nM}$ ) (Table 2). Strong affinity of Enalaprilat for ACE2 suggests that it might prevent the entry of SARS-CoV-2 to human cells. Notably, two of the drug candidates identified, namely Daclatasvir and Ombitasvir, are known Hepatitis C virus (HCV) inhibitors, thus suggesting that they may act against both HCV and SARS-CoV2 (Zahradnik et al., 2021). The DrugBank has collected data for 65 drugs against 385 drug targets, which is web accessible at https://go.drugbank.com/covid-19.

Zeng et al. used deep learning-based knowledge graph to select promising SARS-CoV-2 drug candidates (Zeng et al., 2020). Knowledge graph in this study encompasses 15 million edges across 39 types of relationships connecting expression patterns, genes, pathways, drugs and diseases and incorporates data from over 20 million PubMed articles and the DrugBank database (Table 1). Deep learning employed to learn the representation of nodes and relationships in this knowledge graph led to identification of 41 promising drug candidates, including

${ }^{1}$ https://www.bindingdb.org/bind/Covid19.jsp
Toremifene, Niclosamide, Dexamethasone and Indomethacin (Table 2; Beck et al., 2020). Toremifene is a selective estrogen receptor modulator, which has shown antiviral activity against a number of viruses, including SARS-CoV-2. Dexamethasone is an anti-inflammatory agent with the potential to treat SARSCoV-2 infections (Beck et al., 2020). Niclosamide, a drug used to treat tapeworm and an anti-inflammatory drug Indomethacin were also shown to have antiviral activity in vitro. The 41 promising drug candidates identified in this study (including Toremifene, Niclosamide, Dexamethasone and Indomethacin) were also validated by gene expression and proteomics of cells infected with SARS-CoV-2 (Beck et al., 2020).

A hybrid deep learning and molecular simulation-based screening procedure was used to select drug candidates targeting RNA-dependent RNA polymerase (RdRp) from 1906 approved drugs, recently (Choi et al., 2020). Commercially available drug candidates, Pralatrexate and Azithromycin, (Table 2) identified in this study were confirmed to inhibit SARS-CoV-2 replication in vitro (Choi et al., 2020). While Pralatrexate was shown to act after entry of the virus into the cells, Azithromycin was active at both the entry and post-entry of SARS-CoV-2 into the host cells (Choi et al., 2020).

Bai et al. developed MolAICal software tool combining deep learning model and classical algorithm for identification of drugs interacting with 3D pocket of protein targets (Bai et al., 2020). Deep learning model of MolAICal software was trained using approved drug fragments in PDBbind database and drug-like molecules in the ZINC database (Table 1). Drug design functions of MolAICal software were demonstrated using the membrane protein glucagon receptor (GCGR) and SARS-CoV-2 main protease (Mpro) (Zeng et al., 2020).

Ton et al. (2020) developed a Deep Docking (DD) deep learning platform which uses QSAR models for screening of potential drug candidates in the ZINC database (Table 1). This approach led to the identification of 1,000 potential ligands for SARS-CoV-2 Mpro (Ton et al., 2020).

Deep learning and molecular docking methods were developed for screening of natural compounds against SARSCoV-2 Mpro in the ChEMBL database (Table 1; Bai et al., 2020). ChEMBL database is an open large-scale chemical database of bioactive molecules, containing 8,200 potential anti-SARS$\mathrm{CoV}-2$ drug candidates. This study led to the identification of two natural compounds with potential as therapeutics against SARS-CoV-2, namely Palmatine (Kd 1096.4 nM) and Sauchinone (Table 2) (Kd $389.05 \mathrm{nM}$ ). Palmatine and Sauchinone are an alkaloid and a lignan, respectively, with previously shown pharmacological properties. Furthermore, both Palmatine and Sauchinone form a stable complex with SARS-CoV-2 Mpro and have been predicted to inhibit SARS-CoV-2 (Bai et al., 2020).

Deep learning combined with multiple sequence alignment drug-likeness screening, molecular docking, chemical space mapping and molecular dynamics simulation was also used to identify drug candidates by screening 1528 anti-HIV1 compounds against 3-chymotrypsin-like cysteine protease (3CLpro) of SARS-CoV-2 (Nand et al., 2020).

Given the lack of therapeutics against SARS-CoV-2, deep learning approaches combined with other computational 
TABLE 2 | Drug candidates against SARS-CoV-2.

\begin{tabular}{|c|c|c|c|c|c|}
\hline Drug & $\begin{array}{l}\text { Molecular } \\
\text { formula }\end{array}$ & $\begin{array}{c}\text { Structural } \\
\text { formula }\end{array}$ & SMILES & Target & References \\
\hline Atazanavir & $\mathrm{C}_{38} \mathrm{H}_{52} \mathrm{~N}_{6} \mathrm{O}_{7}$ & & $\begin{array}{c}\mathrm{COC}(\mathrm{N}[\mathrm{C} @ @ \mathrm{H}](\mathrm{C}(\mathrm{C})(\mathrm{C}) \mathrm{C}) \mathrm{C}(\mathrm{NN} \\
(\mathrm{C}[\mathrm{C} @ \mathrm{H}](\mathrm{O})[\mathrm{C} @ \mathrm{H}](\mathrm{CC} 1=\mathrm{CC}= \\
\mathrm{CC}=\mathrm{C} 1) \mathrm{NC}([\mathrm{C} @ \mathrm{H}](\mathrm{C}(\mathrm{C})(\mathrm{C}) \mathrm{C}) \mathrm{NC} \\
(\mathrm{OC})=\mathrm{O})=\mathrm{O}) \mathrm{CC}(\mathrm{C}=\mathrm{C} 2)=\mathrm{CC}= \\
\mathrm{C} 2 \mathrm{C} 3=\mathrm{NC}=\mathrm{CC}=\mathrm{C} 3)=\mathrm{O})=\mathrm{O}\end{array}$ & $\begin{array}{c}\text { 3C-like } \\
\text { proteinase }\end{array}$ & $\begin{array}{l}\text { Beck et al., } \\
2020\end{array}$ \\
\hline $\begin{array}{l}\text { Kaletra } \\
\text { (Lopinavir/Ritonavir) }\end{array}$ & $\begin{array}{c}\mathrm{C}_{74} \mathrm{H}_{96} \mathrm{~N}_{10} \mathrm{O}_{10} \mathrm{~S}_{2} \\
\left(\mathrm{C}_{37} \mathrm{H}_{48} \mathrm{~N}_{4} \mathrm{O}_{5} / \mathrm{C}_{37}\right. \\
\left.\mathrm{H}_{48} \mathrm{~N}_{6} \mathrm{O}_{5} \mathrm{~S}_{2}\right)\end{array}$ & & $\begin{array}{c}\mathrm{CC} 1=\mathrm{CC}=\mathrm{CC}(\mathrm{C})=\mathrm{C} 10 \mathrm{CC}(\mathrm{N} \\
[\mathrm{C} @ @]](\mathrm{CC} 2=\mathrm{CC}=\mathrm{CC}=\mathrm{C} 2) \\
[\mathrm{C} @ @]](\mathrm{O}) \mathrm{C}[\mathrm{C} @ \mathrm{H}](\mathrm{CC} 3=\mathrm{CC} \\
=\mathrm{CC}=\mathrm{C} 3) \mathrm{NC}([\mathrm{C} @ \mathrm{H}](\mathrm{C}(\mathrm{C}) \mathrm{C}) \\
\mathrm{N} 4 \mathrm{C}(\mathrm{NCCC} 4)=\mathrm{O})=\mathrm{O})=\mathrm{O}\end{array}$ & Helicase & $\begin{array}{l}\text { Beck et al., } \\
2020\end{array}$ \\
\hline Enalaprilat & $\mathrm{C}_{18} \mathrm{H}_{24} \mathrm{~N}_{2} \mathrm{O}_{5}$ & & $\begin{array}{l}\mathrm{OC}([\mathrm{C} @ \mathrm{H}] 1 \mathrm{~N}(\mathrm{C}([\mathrm{C} @ \mathrm{H}](\mathrm{C}) \mathrm{N} \\
{[\mathrm{C} @ \mathrm{H}](\mathrm{C}(\mathrm{O})=\mathrm{O}) \mathrm{CCC} 2=\mathrm{CC}} \\
=\mathrm{CC}=\mathrm{C} 2)=\mathrm{O}) \mathrm{CCC} 1)=\mathrm{O}\end{array}$ & ACE2 & $\begin{array}{l}\text { Choi et al., } \\
2020\end{array}$ \\
\hline Venetoclax & C45H50CIN7O7S & & $\begin{array}{c}\mathrm{ClC} 1=\mathrm{CC}=\mathrm{C}(\mathrm{C} 2=\mathrm{C}(\mathrm{CN} 3 \mathrm{CC} \\
\mathrm{N}(\mathrm{C} 4=\mathrm{CC}=\mathrm{C}(\mathrm{C}(\mathrm{NS}(\mathrm{C} 5=\mathrm{CC}= \\
\mathrm{C}(\mathrm{NCC} 6 \mathrm{CCOCC}) \mathrm{C}([\mathrm{N}+]([\mathrm{O}- \\
])=\mathrm{O})=\mathrm{C} 5)(=\mathrm{O})=\mathrm{O})=\mathrm{O}) \mathrm{C}(\mathrm{OC} \\
7=\mathrm{CC}(\mathrm{C}=\mathrm{CN} 8)=\mathrm{C} 8 \mathrm{~N}=\mathrm{C} 7)= \\
\mathrm{C} 4) \mathrm{CC} 3) \mathrm{CCC}(\mathrm{C})(\mathrm{C}) \mathrm{C} 2) \mathrm{C}=\mathrm{C} 1\end{array}$ & $\begin{array}{l}\text { TMPRSS2 } \\
\text { ACE2 }\end{array}$ & $\begin{array}{l}\text { Choi et al., } \\
2020\end{array}$ \\
\hline Ombitasvir & $\mathrm{C}_{50} \mathrm{H}_{67} \mathrm{~N}_{7} \mathrm{O}_{8}$ & & $\begin{array}{c}\mathrm{CC}(\mathrm{C})(\mathrm{C}) \mathrm{C}(\mathrm{C}=\mathrm{C} 1)=\mathrm{CC}=\mathrm{C} 1 \mathrm{~N}([\mathrm{CC} @ \\
\mathrm{H}](\mathrm{C} 2=\mathrm{CC}=\mathrm{C}(\mathrm{NC}([\mathrm{C} @ @ \mathrm{H}] 3 \mathrm{CCCN} \\
3 \mathrm{C}([\mathrm{C} @ \mathrm{H}](\mathrm{C}(\mathrm{C}) \mathrm{C}) \mathrm{NC}(\mathrm{OC})=\mathrm{O})=\mathrm{O}) \\
=\mathrm{O}) \mathrm{C}=\mathrm{C} 2) \mathrm{CC} 4)[\mathrm{C} @ @ \mathrm{H}] 4 \mathrm{C} 5=\mathrm{CC} \\
=\mathrm{C}(\mathrm{NC}([\mathrm{C} @ \mathrm{H}] 6 \mathrm{~N}(\mathrm{C}([\mathrm{C} @ @ \mathrm{H}](\mathrm{NC}(\mathrm{O} \\
\mathrm{C})=\mathrm{O}) \mathrm{C}(\mathrm{C}) \mathrm{C})=\mathrm{O}) \mathrm{CCC} 6)=\mathrm{O}) \mathrm{C}=\mathrm{C} 5\end{array}$ & $\begin{array}{l}\text { TMPRSS2 } \\
\text { ACE2 }\end{array}$ & $\begin{array}{l}\text { Choi et al., } \\
2020\end{array}$ \\
\hline Toremifene & $\mathrm{C}_{26} \mathrm{H}_{28} \mathrm{CINO}$ & & $\begin{array}{c}\mathrm{CN}(\mathrm{C}) \mathrm{CCOC} 1=\mathrm{CC}=\mathrm{C}(/ \mathrm{C}(\mathrm{C} 2 \\
=\mathrm{CC}=\mathrm{CC}=\mathrm{C} 2)=\mathrm{C}(\mathrm{C} 3=\mathrm{C} \\
\mathrm{C}=\mathrm{CC}=\mathrm{C} 3) / \mathrm{CCCl}) \mathrm{C}=\mathrm{C} 1\end{array}$ & - & $\begin{array}{l}\text { Zeng et al., } \\
2020\end{array}$ \\
\hline
\end{tabular}


TABLE 2 | (Continued)

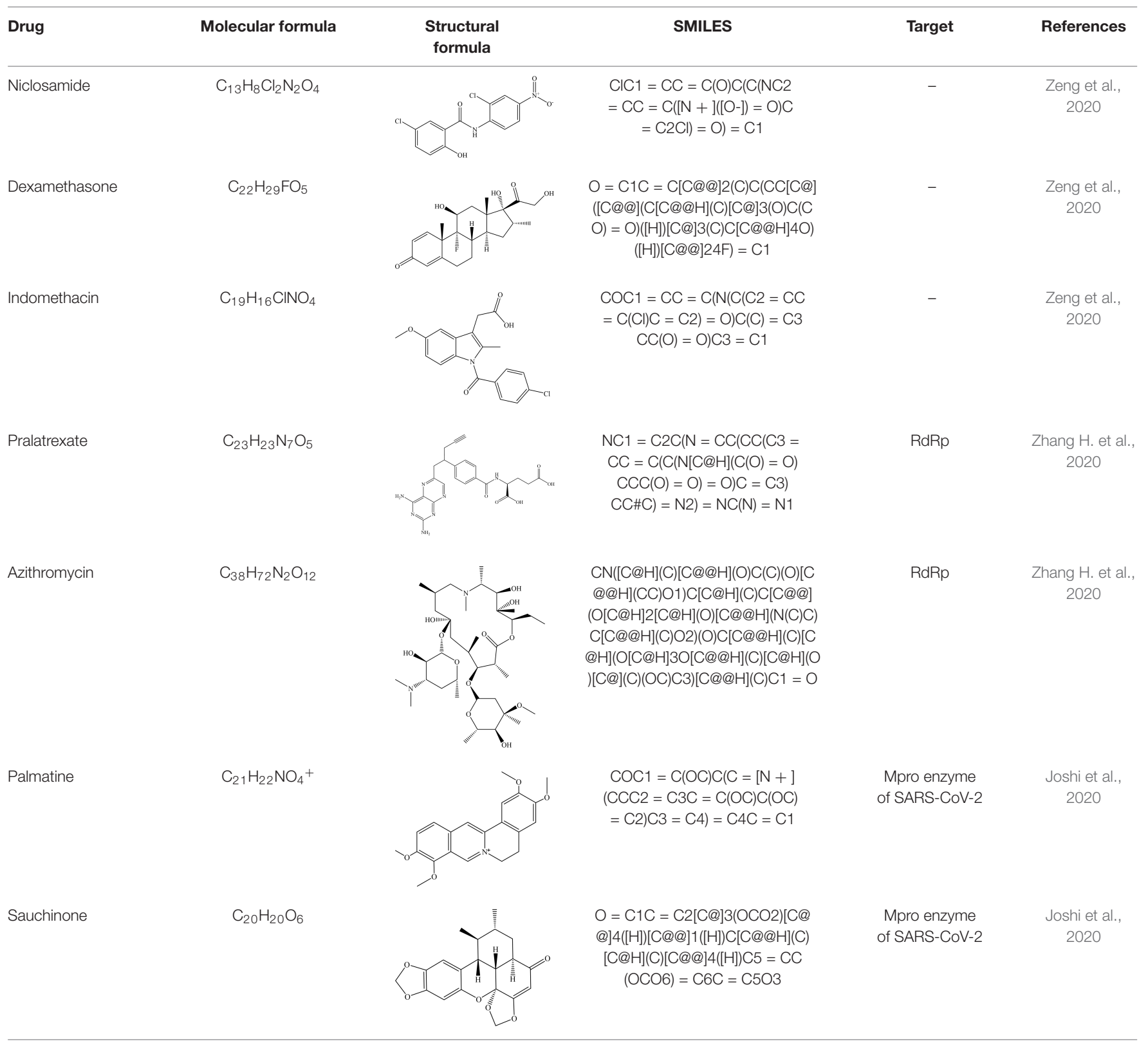

Table shows molecular and structural formulas, simplified molecular-input line-entry system (SMILES) strings and corresponding targets of the potential drugs against SARS-COV-2.

methods will play an important role in the identification of potential drugs targeting SARS-CoV-2. Compounds selected by deep learning will subsequently undergo standard clinical evaluation.

\section{DISCUSSION}

Deep learning has a number of advantages compared to more conventional methods, including its ability to learn complex features independently. Although deep learning has played an important role in the identification of novel drugs against a wide range of pathogens, including SARS-CoV-2, many challenges still remain.

The connection between the data fed into the deep learning model and the delivered output is inscrutable, which hidden inside is a so-called black box. Deep neural network due to its black-box nature therefore often lacks interpretability. Therefore, the interpretability of the future neural networks on the output results will be a key factor in understanding the logic of machine. This will aid analysis of the chemical compounds identified by deep learning and better design of the drug discovery studies.

Furthermore, the input data affects the prediction performance of the deep learning model. Consequently, a large, 
standardized and reliable biochemical dataset is necessary to achieve better learning of the deep learning model. Development of a large open dataset in the future will enable potential standardization of the deep learning-based drug discovery.

Antibody-based therapy represents an interesting SARS-CoV2 treatment option. Deep learning models have been developed for the discovery and design of therapeutic antibodies (Mason et al., 2021; Saka et al., 2021). Thus, drug repositioning and screening from computational libraries containing a massively diverse antibody sequences could be used to engineer anti-viral SARS-CoV-2 treatment.

Furthermore, most recent studies describe methodologies separately and test them individually. Application of deep learning to combine chemoinformatics with other types of data, such as imaging, cellular and molecular biology data for integrative analysis would be an important direction for future research. To this end, it might be necessary to identify the best neural network architecture for handling those vast troves of data.

We believe that integrative and systematic analysis will be important for future deep learning-based drug discovery that

\section{REFERENCES}

Andrianov, A. M., Nikolaev, G. I., Shuldov, N. A., Bosko, I. P., Anischenko, A. I., and Tuzikov, A. V. (2021). Application of deep learning and molecular modeling to identify small drug-like compounds as potential HIV-1 entry inhibitors. J. Biomol. Struct. Dyn. 15, 1-19. doi: 10.1080/07391102.2021. 1905559

Bai, Q., Tan, S., Xu, T., Liu, H., Huang, J., and Yao, X. (2020). MolAICal: a soft tool for $3 \mathrm{D}$ drug design of protein targets by artificial intelligence and classical algorithm. Brief Bioinform. 11:bbaa161. doi: 10.1093/bib/bb aa161

Beck, B. R., Shin, B., Choi, Y., Park, S., and Kang, K. (2020). Predicting commercially available antiviral drugs that may act on the novel coronavirus (SARS-CoV-2) through a drug-target interaction deep learning model. Comput. Struct. Biotec. 18, 784-790. doi: 10.1016/j.csbj.2020. 03.025

Bender, A., Scheiber, J., Glick, M., Davies, J. W., Azzaoui, K., Hamon, J., et al. (2007). Analysis of pharmacology data and the prediction of adverse drug reactions and off-target effects from chemical structure. ChemMedChem 2, 861-873. doi: $10.1002 / \mathrm{cmdc} .200700026$

Chen, H., Engkvist, O., Wang, Y., Olivecrona, M., and Blaschke, T. (2018). The rise of deep learning in drug discovery. Drug Discov. Today 23, 1241-1250. doi: 10.1016/j.drudis.2018.01.039

Choi, Y., Shin, B., Kang, K., Park, S., and Beck, B. R. (2020). Target-centered drug repurposing predictions of Human Angiotensin-Converting Enzyme 2 (ACE2) and Transmembrane Protease Serine Subtype 2 (TMPRSS2) interacting approved drugs for Coronavirus disease 2019 (COVID-19) treatment through a drug-target interaction deep learning model. Viruses 12:1325. doi: 10.3390/ v12111325

Duch, W., Swaminathan, K., and Meller, M. (2007). Artificial intelligence approaches for rational drug design and discovery. Curr. Pharm. Des. 13, 1497-1508. doi: 10.2174/138161207780765954

He, B., Huang, J., and Chen, H. (2019). PVsiRNAPred: Prediction of plant exclusive virus-derived small interfering RNAs by deep convolutional neural network. J. Bioinform. Comput. Biol. 17:1950039. doi: 10.1142/S02197200195 00392

Joshi, T., Joshi, T., Pundir, H., Sharma, P., Mathpal, S., and Chandra, S. (2020). Predictive modeling by deep learning, virtual screening and molecular dynamics study of natural compounds against SARS-CoV-2 main protease. J. Biomol. Struct. Dyn. 5, 1-19. doi: 10.1080/07391102.2020.18 02341 involves complicated large biological, chemical and clinical datasets. Using such large datasets to streamline and accelerate drug discovery, deep learning will be crucial not only for the identification of drug candidates against SARS-CoV-2 but also against a broad spectrum of other emerging and reemerging pathogens.

\section{AUTHOR CONTRIBUTIONS}

All authors contribute to the writing and reviewing the manuscript.

\section{FUNDING}

This work was supported by the Natural Science Foundation of Shenzhen City (Project No. JCYJ20180306172131515) and the Shenzhen Science and Technology Program the university stable support program (20200821222112001).

Li, J., Pu, Y., Tang, J., Zou, Q., and Guo, F. (2020). DeepAVP: A dualchannel deep neural network for identifying variable-length antiviral peptides. IEEE J. Biomed. Health Inform. 24, 3012-3019. doi: 10.1109/JBHI.2020.2 977091

Lipinski, C. F., Maltarollo, V. G., Oliveira, P. R., daSilva, A. B. F., and Honorio, K. M. (2019). Advances and Perspectives in applying deep learning for drug design and discovery. Front. Robot. 6:108. doi: 10.3389/frobt.2019.0 0108

Mason, D. M., Friedensohn, S., Weber, C. R., Jordi, C., Wagner, B., Meng, S., et al. (2021). Optimization of therapeutic antibodies by predicting antigen specificity from antibody sequence via deep learning. Nat. Biomed. Eng. 5, 600-612. doi: 10.1038/s41551-021-00699-9

Nand, M., Maiti, P., Joshi, T., and Chandra, S. (2020). A Ramakrishnan Virtual screening of anti-HIV1 compounds against SARS-CoV-2: machine learning modeling, chemoinformatics and molecular dynamics simulation based analysis. Sci. Rep. 10:20397. doi: 10.1038/s41598-020-7 7524-x

Neves, B. J., Braga, R. C., Alves, V. M., Lima, M. N. N., Cassiano, G. C., Muratov, E., et al. (2020). Deep Learning-driven research for drug discovery: Tackling Malaria. PLoS Comput. Biol. 16:e1007025. doi: 10.1371/journal.pcbi.10 07025

Saka, K., Kakuzaki, T., Metsugi, S., Kashiwagi, D., Yoshida, K., Wada, M., et al. (2021). Antibody design using LSTM based deep generative model from phage display library for affinity maturation. Sci. Rep. 11:5852. doi: 10.1038/s41598021-85274-7

Stephenson, N., Shane, E., Chase, J., Rowland, J., Ries, D., Justice, N., et al. (2019). Survey of machine learning techniques in drug discovery. Curr. Drug Metab. 20, 185-193. doi: 10.2174/138920021966618082 0112457

Stokes, J. M., Yang, K., Swanson, K., Jin, W. G., Cubillos-Ruiz, A., Donghia, N. M., et al. (2020). A deep learning approach to antibiotic discovery. Cell 180, 688-702 doi: 10.1016/j.cell.2020 .01 .021

Timmons, P. B., and Hewage, C. M. (2021). ENNAVIA is a novel method which employs neural networks for antiviral and anti-coronavirus activity prediction for therapeutic peptides. Brief Bioinform. 2021:bbab258. doi: 10.1093/bib/ bbab258

Ton, A. T., Gentile, F., Hsing, M., Ban, F., and Cherkasov, A. (2020). Rapid identification of potential inhibitors of SARS $-\mathrm{CoV}-2$ main protease by deep docking of 1.3 billion compounds. Mol. Inform. 8:e2000028. doi: 10.1002/minf. 202000028 
Zahradnik, J., Marciano, S., Shemesh, M., Zoler, E., Harari, D., Chiaravalli, J., et al. (2021). SARS-CoV-2 variant prediction and antiviral drug design are enabled by RBD in vitro evolution. Nat. Microbiol. 6, 1188-1198. doi: 10.1038/s41564021-00954-4

Zeng, X., Song, X., Ma, T., Pan, X., Zhou, Y., Hou, Y., et al. (2020). Repurpose open data to discover therapeutics for COVID-19 using deep learning. J. Proteome. Res. 19, 4624-4636. doi: 10.1021/acs.jproteome.0 c00316

Zhang, H., Yang, Y., Li, J., Wang, M., Saravanan, K. M., Wei, J., et al. (2020). A novel virtual screening procedure identifies Pralatrexate as inhibitor of SARS-CoV-2 RdRp and it reduces viral replication in vitro. PLoS Comput. Biol. 16:e1008489. doi: 10.1371/journal.pcbi.1 008489

Zhang, Y., Xi, H., and Juhas, M. (2020). Biosensing detection of the SARSCoV-2 D614G mutation. Trends Genet. 37, 299-302. doi: 10.1016/j.tig.2020.1 2.004
Conflict of Interest: The authors declare that the research was conducted in the absence of any commercial or financial relationships that could be construed as a potential conflict of interest.

Publisher's Note: All claims expressed in this article are solely those of the authors and do not necessarily represent those of their affiliated organizations, or those of the publisher, the editors and the reviewers. Any product that may be evaluated in this article, or claim that may be made by its manufacturer, is not guaranteed or endorsed by the publisher.

Copyright () 2021 Zhang, Ye, Xi, Juhas and Li. This is an open-access article distributed under the terms of the Creative Commons Attribution License (CC BY). The use, distribution or reproduction in other forums is permitted, provided the original author(s) and the copyright owner(s) are credited and that the original publication in this journal is cited, in accordance with accepted academic practice. No use, distribution or reproduction is permitted which does not comply with these terms. 